\title{
Stability Analysis of the IBR-2M Pulsed Reactor in Automatic Regulated Regime at Various Levels of Average Power
}

\author{
Yuriy N. Pepelyshev ${ }^{1}$, Alexander K. Popov ${ }^{1}$, and Sumkhuu Davaasuren ${ }^{1,2, \star}$ \\ ${ }^{1}$ Frank Laboratory of Neutron Physics, Joint Institute for Nuclear Research, Dubna, Russia \\ ${ }^{2}$ Institute of Physics and Technology, Mongolian Academy of Sciences, Ulaanbaatar, Mongolia
}

\begin{abstract}
The IBR-2M pulse reactor is characterized by a high level of reactivity fluctuations and consequently by a high level of fluctuations in the regulated parameter as well as in the amplitude of the power pulses. Fluctuations of the regulated parameter relative to the average level in the standard stabilization regime usually fall within the range of $20 \%$ with certain peaks up to $40 \%$, which is close to the limits of the emergency protection operation (50\%). Under these conditions the automatic regulator may receive contradictory requirements. In this paper, the results of stability analysis of the IBR-2M reactor in the automatic regulating regime at various levels of average power are presented. It is shown that stability margins of the amplitude and the phase are enough, especially for the IBR-2M reactor operation with automatic regulator.
\end{abstract}

\section{Introduction}

In contrast to stationary reactors characterized by a constant neutron flux, the IBR-2M reactor generates periodic neutron pulses. The IBR-2M reactor generates short neutron pulses ( $200 \mu \mathrm{s}$ at half width) with period $0.2 \mathrm{~s}$ and amplitude of $1830 \mathrm{MW}$. In the IBR-2M reactivity pulses are produced by the reactivity modulators rotating near the core. When the two reflectors pass the core simultaneously, a reactivity pulse develops and for a short time $(450 \mu \mathrm{s})$ the reactor stops being in a super-critical state with prompt neutrons. As the reflectors move away from the reactor core, the reactor becomes deeply sub-critical. The controlled parameter of the reactor is the energy deviation of the power pulse $E_{p}$ from its basic value $E_{p}^{0}$ in relative units $\left(\Delta e_{p}=\left(E_{p}-E_{p}^{0}\right) / E_{p}^{0}\right)[1]$. The basic value is the average amplitude corresponding to the stationary regime, i.e., the regime in which the reactor average power is generally constant [2]-[4].

The IBR-2M reactor is equipped with a power automatic regulator (AR). The principal aim of the AR is to automatically bring the reactor to the average power preset level and to stabilize the reactor power at the preset level. The structure and parameters of the AR have essential influence on the power transition processes. At the stage of IBR-2M design, an algorithm of the AR reactivity forming was derived. The algorithm provided a statistically efficient power level, i.e., the attainment of a minimum of the mean-square energy deviation for the future power pulse based on information obtained from the previous pulses [5]. It was assumed when deriving the AR algorithm that random reactivity excitations with a normal distribution law affects the reactor. It was as well assumed that the

\footnotetext{
${ }^{\star}$ e-mail: dsumkhuu@jinr.ru
} 
AR estimates the reactivity values corresponding to previous power pulses with a certain statistical measurement error which is also characterized by a normal distribution law. In addition, a concept of the information "aging" rate in the AR was introduced. It was shown as a result that the AR must represent an integrating element to the input of which energy deviation of the current power pulse from its base value is applied. The conventional AR of the IBR-2M, besides the regulation rod with the step motor and its control unit, includes also the smoothing unit. The output signal of the smoothing unit arrives to the control unit of the step motor and is shaped by smoothing power pulse energy deviation relatively to the base value. The smoothing unit is a time-lag element and the step motor with the regulation rod and control unit form the integrating element [6].

In this paper, results of a study of the influence of the AR parameters values $(\Delta=0.05,0.1,0.15$, 0.2 is the parameter of the step motor velocity $\mathrm{AR}$ and $q=1,2, \ldots, 32$ is the smoothing coefficient) on the stability of the IBR-2M reactor in automatic regime of operation are reported.

\section{Stability analysis of the IBR-2M reactor operation with the automatic regulator}

The model of the IBR-2M reactor is designed to simulate the transient processes, calculate the frequency characteristics of the reactor for the system as a whole and the constituent components, and assess the stability of the reactor. If a reactor operating at power undergoes a small perturbation in reactivity, however, it is possible to linearize the equations for the point reactor [7]. The IBR-2M reactor with automatic regulator is represented as a linearized closed single-loop system with negative feedback (Fig. 1). The reactivity deviation $\Delta r_{n}$ is taken as the input signal of the closed system, and the relative deviation $\Delta e_{p n}$ of the energy of the power pulses from the basic value is taken as the output signal. The main dynamic properties of a pulsed reactor can be determined if its amplitudephase-frequency characteristics (APFCs), or Nyquist plots, are known. The APFC is a plot of the pulse frequency transfer function of a reactor $W(j \omega)=U(\omega)+j V(\omega)$ on the complex plane.

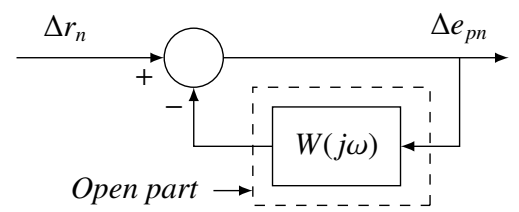

Figure 1. A linearized model of IBR-2M in the self-regulation regime, represented as a single-loop closed system

The pulse transfer function $W(j \omega)$ is given by

$$
W(j \omega)=\frac{W_{A}(j \omega)}{1+\frac{1-k_{b}}{1-k_{b} \sum_{i=1}^{6} W_{s i}(j \omega)}\left[E^{0} \sum_{j=1}^{3} W_{T j}(j \omega)-\sum_{i=1}^{6} W_{s i}(j \omega)\right]},
$$

where $k_{b}$ and $E^{0}$ are a constant parameters [1]. $W_{A}(j \omega), W_{s i}(j \omega)$ and $W_{T j}(j \omega)$ denote the transfer functions of the automatic regulator, the $i$-th delayed neutrons group and the $j$-th power feedback component, respectively.

Here, $W_{A}(j \omega)$ denotes the $\Delta$ and $q$ dependent transfer function of the AR which is given by

$$
W_{A}(j \omega)=\frac{v_{\Delta} m_{A} T_{p}}{\Delta} \frac{\exp (-j \omega)}{[q-(q-1) \exp (-j \omega)][1-\exp (-j \omega)]},
$$

where $v_{\Delta} m_{A}=0.08 \cdot 1.54 \cdot 10^{-4} \mathrm{~s}^{-1}$ is a constant factor, $T_{p}=0.2 \mathrm{~s}$ is the period of the power pulse, $\Delta$ is the parameter of the automatic regulator speed $(0.05,0.1,0.15$ or 0.2$)$ and $q$ is the smoothing coefficient of the automatic regulator $(1,2, \ldots, 32)$. 
Further, $W_{s i}(j \omega)$ denotes the transfer function of the $i$-th delayed neutrons group

$$
W_{s i}(j \omega)=\frac{\mu_{i} \lambda_{i}}{\sum_{i=1}^{6} \mu_{i} \lambda_{i} \frac{1}{\exp \left(\lambda_{i} T_{p}\right)}} \frac{\exp (-j \omega)}{\exp \left(\lambda_{i} T_{p}\right)-\exp (-j \omega)},
$$

where, $\mu_{i}$ and $\lambda_{i}$ denote the delayed neutron fraction and the decay constant of the source of $i$-th delayed neutrons group, respectively [8].

Finally, $W_{T j}(j \omega)$ denotes the transfer function of the $j$-th component of the power feedback:

$$
W_{T j}(j \omega)=\frac{k_{T j}}{T_{T j}} \frac{\exp (-j \omega)}{\exp \left(T_{p} / T_{T j}\right)-\exp (-j \omega)},
$$

where $k_{T j}$ is the transfer coefficient and $T_{T j}$ is the time constant of the $j$-th components of the power feedback. The parameters of power feedback, which are introduced into the model of the reactor dynamics, were determined by analyzing experimental transients of power using a special search algorithm [9].
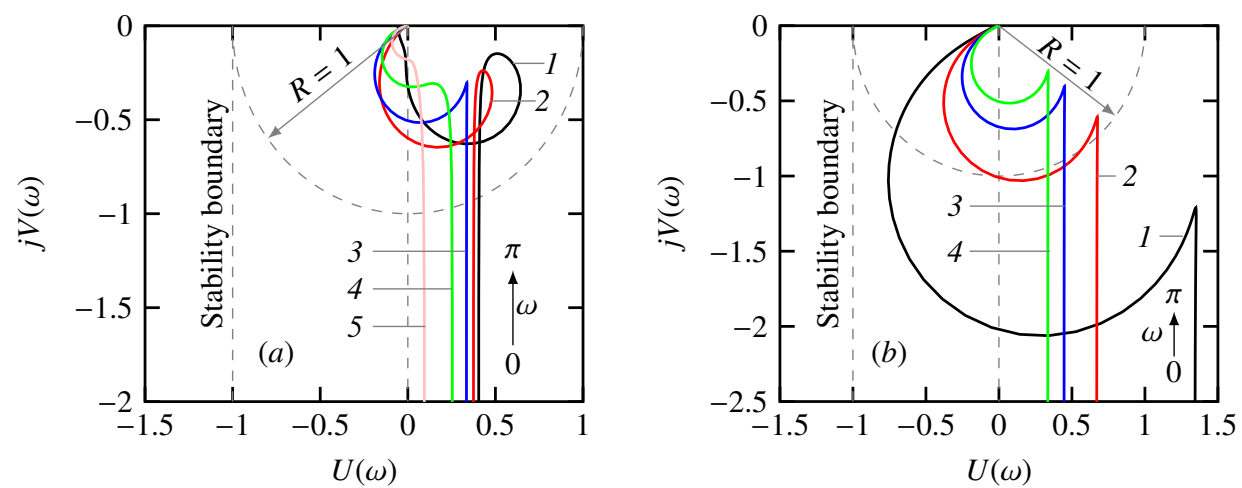

Figure 2. The amplitude-phase frequency characteristic of the open part of the IBR-2M reactor in the automatic regulated regime at $2 \mathrm{MW}$ of average power. $a) \Delta=0.2$ and $q=1(1), 4(2), 8(3), 16(4), 32(5), b) q=8$ and $\Delta=0.05(1), 0.1(2), 0.15(3), 0.2(4)$.

The oscillation frequency in the pulse transfer function $W(j \omega)$ varies from 0 to $0.5 f_{p}$, where $f_{p}=1 / T_{p}$ is the power pulse frequency, and the dimensionless frequency $\omega=2 \pi f_{p} T_{p}$ varies from 0 to $\pi$. The length of the vector from the origin of coordinates to the point of frequency $\omega(|W(j \omega)|)$ is the amplitude-frequency characteristic (gain), and the angle of rotation of the vector from the real positive semi axis $\varphi(\omega)=\arctan [V(\omega) / U(\omega)]$ is the phase-frequency characteristic of the reactor. For example, for the pulsed reactor APFC (this characteristic will be considered in more detail below, Fig. 2) the length of the vector drawn from the origin to any point on the curve corresponding to a frequency $\omega$ is equal to the ratio between the amplitude of the sine power pulse energy oscillation in relative units of $\Delta e_{p}=\left(E_{p}-E_{p}^{0}\right) / E_{p}^{0}$ (output value) and the amplitude of the sine reactivity oscillation (input value) expressed in terms of parts of $\beta_{p}$. Here $E_{p}$ and $E_{p}^{0}$ are the energy of the current power pulse and its basic value. The angle of rotation of the vector with respect to the real positive semi axis is a phase shift between the power and reactivity oscillations.

The assessment of the reactor stability using the Nyquist stability criterion is done as follows. The reactor is represented as a closed one-loop system. While the open system is stable or neutral, the closed system is stable provided that the APFC of the open system does not include the point with the coordinates $(-1,0)$. The amplitude (or gain) stability margin $(a)$ shows how many times this 
coefficient must be increased to bring the system from the stable state to the stability boundary $(-1,0)$. The phase margin $(\varphi)$ is defined as the angle of rotation of the unit-length vector at which its end turns out to be at the point with the coordinates $(-1,0)$ and the APFC of the open system goes through this point, i.e., the closed system reaches the stability boundary. In the Fig. 2 the phase margin at the different values of the AR parameters is defined as the dashed unit circle. The phase margin reflects possible influence of the additional neglected inertia of the system.

\section{Conclusions}

An analysis was reported of the amplitude and phase frequency characteristics of the open part of the closed loop system which corresponds to the IBR-2M reactor operation in the automatic regulated regime. Estimated stability margins of the amplitude $(a)$ and phase $(\varphi)$ of the reactor IBR-2M at different level of average power have been found. In Fig. 3 the dependence of the stability margins on the parameters of $\operatorname{AR}(\Delta$ - parameter of the step motor velocity $\operatorname{AR}$ and $q$ - smoothing coefficient) at the average power $2 \mathrm{MW}$ are shown. It is shown that the stability margins of the amplitude and the phase are well within the safety regime, especially for the automatic regulated regime, characterized by $\Delta=0.05$ and $4<q<20$ at $2 \mathrm{MW}$ of average power.
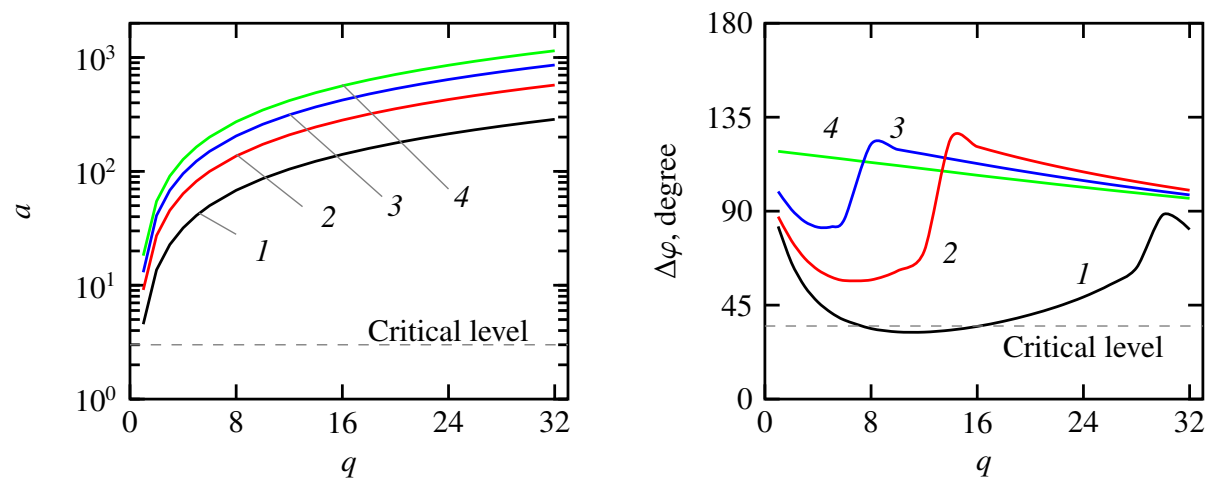

Figure 3. Dependence of the stability margins of the amplitude (left) and the phase (right) on the parameters values $(q$ and $\Delta)$ of the automatic regulator of the IBR-2M reactor at the average power of $2 \mathrm{MW}$. The labels 1 , $2,3,4$ point to curves obtained for $\Delta=0.05, \Delta=0.1, \Delta=0.15$, and $\Delta=0.2$, respectively.

\section{References}

[1] Yu.N. Pepelyshev, A.K. Popov, and D. Sumkhuu, Annals of Nuclear Energy 85, 488-493 (2015)

[2] Yu.N. Pepelyshev, A.K. Popov, D. Sumkhuu, and D. Sangaa, Physics of Particles and Nuclei Letters 12 (3), 435-438 (2015)

[3] E.A. Bondarchenko, Yu.N. Pepelyshev, and A.K. Popov, Fiz. Elem. Chastits At. Yadra 35 (4), 928-983 (2004)

[4] A.K. Popov, Fundamentals of Nuclear Reactor Control (in Russian, Mosk. Gos. Univ., Moscow, 2012)

[5] A.K. Popov, Atomic Energy 31 (3), p. 269 (1971)

[6] E.A. Bondarchenko, Yu.N. Pepelyshev, and A.K. Popov, Annals of Nuclear Energy 28 (1), 63-78 (2001)

[7] G.J. Bell and S. Glasstone, Nuclear reactor theory (Van Nostrand Reinhold Company, 1970)

[8] G.R. Keepin, Physics of Nuclear Kinetics (Addison-Wesley, 1965)

[9] Yu.N. Pepelyshev, A.K. Popov, and D. Sumkhuu, Atomic Energy 122 (2), 63-67 (2017) 